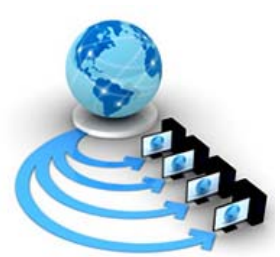

International Journal of Advanced Research in Computer Science

RESEARCH PAPER

\author{
Available Online at www.ijarcs.info
}

\title{
SUPERPIXEL SEGMENTATION BASED DIGITAL IMAGE WATERMARKING FOR COLOR IMAGE AUTHENTICATION
}

\author{
Nirali Jani \\ B.M.College of Computer Application \\ BMEF \\ Surat, India
}

\author{
Dr.Ashish Jani \\ Department of computer Engineering \\ P.P.Savani University \\ Kosamaba, India
}

\begin{abstract}
With thousands of images piling inside the world serves daily, authentication of any of any image at any given time around the world continues to be an undeniable issue. Mounting rise in data and information exchange, a heavy use of images has become common place. The challenges related to image authentication are increasing at an alarming rate. Digital image segmentations in watermarking has been found to be of good use in improve the performance of image authentication. Undertaken work in this paper compare time duration of segments and block based decomposition, watermark self-embedding, segment level watermark embedding and superpixel SVD based watermark embedding approach for image authentication. With this experiments and analysis, the superpixel based image segmentation with SVD has been applied to retain the quality of image of the embedment of digital watermark with acceptable PSNR value. Also time obtain for segmentation is less compare to block based method. The applied work is likely to have its prospective application in medical image segmentation, copyright protection and in digital forensic image investigation.
\end{abstract}

Keywords: Superpixel segmentation, svd, PSNR, self-embedding, image authentication

\section{INTRODUCTION}

With the increasing image data repository volume issues related to authentication of digital assets are increasing. For speed up processing and improvement of results, instead of block and pixel based method proposed scheme use superpixel segmentation approach for watermarking. Majority block based schemes divides whole images into blocks without knowing its color intensity of pixels. Because of this sometime it fails to extract watermark and cannot protect from some attacks. Also decrease tampers detection for authentication [1]. Having identified optimum method as SLIC for superpixel segmentation [2, 3]. For the embedment of watermark three methods self-embedding, individual segment embedding and Superpixel based SVD have been used and SP-SVD method as justified as superiority over other two approaches $[1,4,5]$.

\section{RELATED WORK}

In some presented work, superpixel region used for watermark self-region embedding using chaotic system is proposed. Superpixel content is embedded into another neighbor Superpixel region. Superpixel segments boundaries are marked for extraction process and detect tampered region. Author used Lsb method to embed the watermark into the image [1, 3].Other method presents block svd for embedding color watermark into color host image and through Arnold transformation generate scramble image for security against some attacks like cropping [5]. Here each blocks maximum singular value taken from RGB image block. Using image Singular Values, watermarked image is generated. This scheme is effective for some of the attacks. This scheme is not survive with large angle of rotation. Some other block wise and content wise semi-fragile scheme for authentication and tamper finding. Here author segmented image as per Region of Interest of image with watermark sequence bits and use DCT for embedding in middle frequency band of ROE [6].Compare watermark sequence and extracted sequence from ROE if match then it is authenticated or its tampered. Only grayscale images are experimented.

Image self-embedding adjacent block based method for find the manipulated blocks and authentication of image. Author used statistical detection method for analysis of image authentication and tamper under some content tampering attacks [7].

In present watermarking methods image is divided into same size blocks and watermark image is embed in each block so here image colors contents are ignored [1,4,5]. For more strengthen algorithm, consider image color content during image segmentation and watermark embedding is the issue of the under taken work.

\section{PROPOSED WORK}

The proposed work includes the more precise the segmentation of image by optimization of segmentation time implementing DCT DWT and SLIC.

According to minimum segmentation time, selection of approach SLIC taken for watermark embedding into cover image [2, 3]. The optimum output is then taken to the process of embedment of the watermark to the segments. In further process with the SLIC which consider contents of image during segmentation, three watermark embedding methods are used, Superpixel Self-embedding watermarking Scheme; Superpixel individual segment watermark embedding and Superpixel SVD based embedding method to enhance the Quality of resultant watermarked image.

The work is extended to extract the watermark from image individual segments and get the original image separated with an accuracy attained. 


\section{METHODOLOGY:}

For digital image watermarking, the image is to be decomposing into blocks. For the process of decomposition this work is used DCT DWT and SLIC approaches.

The time needed to decompose image into segment/blocks is measured for optimum position time and the approach to be undertaken in succession.

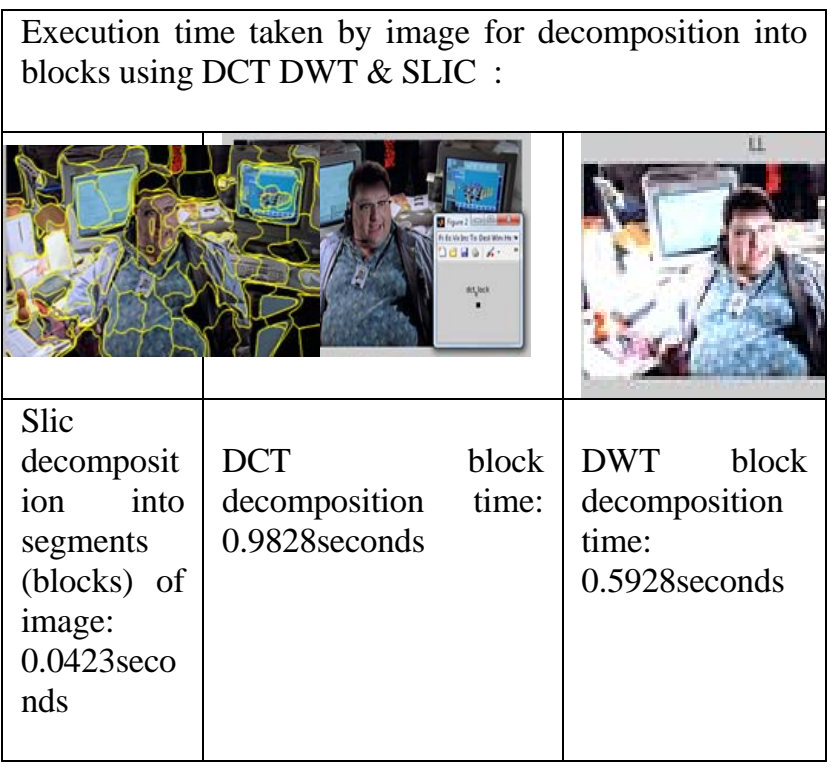

Figure1. Image segmentations using SLIC DCT DWT:

The analysis of the measure time of the decomposition of the image reveals that Slic method is suitable for making Superpixel segments. A Superpixel image segmentation method is used for embedment of watermark into the individual image segments. It can be optimally achieved by Slic method.

The embedment of digital watermark is carried out on the segments. After embedment the components are united to form the resultant image.

This resultant image is compared with original source image by measuring parameter PSNR. The measure value is helping us to ascertain the quality of resultant image.

The analysis of the measure PSNR of the composite image reveals that the PSNR value is $10.45 \mathrm{DB}$ which is far less then acceptable level.

This experiment suggests that by try and error methods different approaches of embedment of watermark into cover image is to be applied so that PSNR value is comes closer to acceptable level.

The extension of the work is carried out with the application of

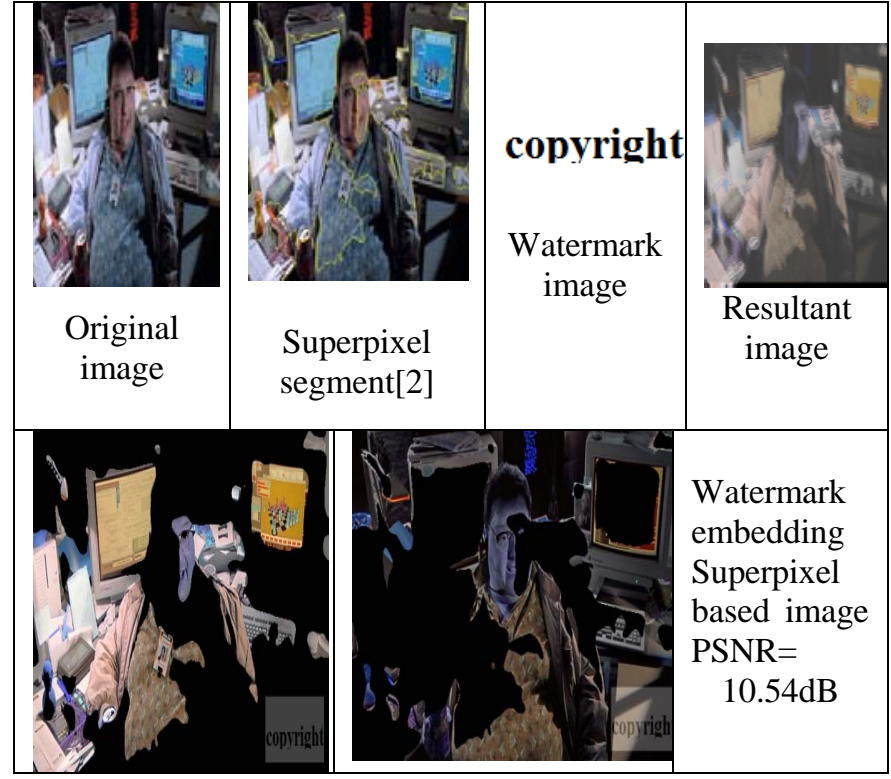

Figure2. Embedding watermark into individual Superpixel segments:

Self-embedding method. In this approach the external watermark is not taken as watermark from within a component is embedded in a component. In this case also PSNR is measure. This approach is indicating increase of marginal value of PSNR.

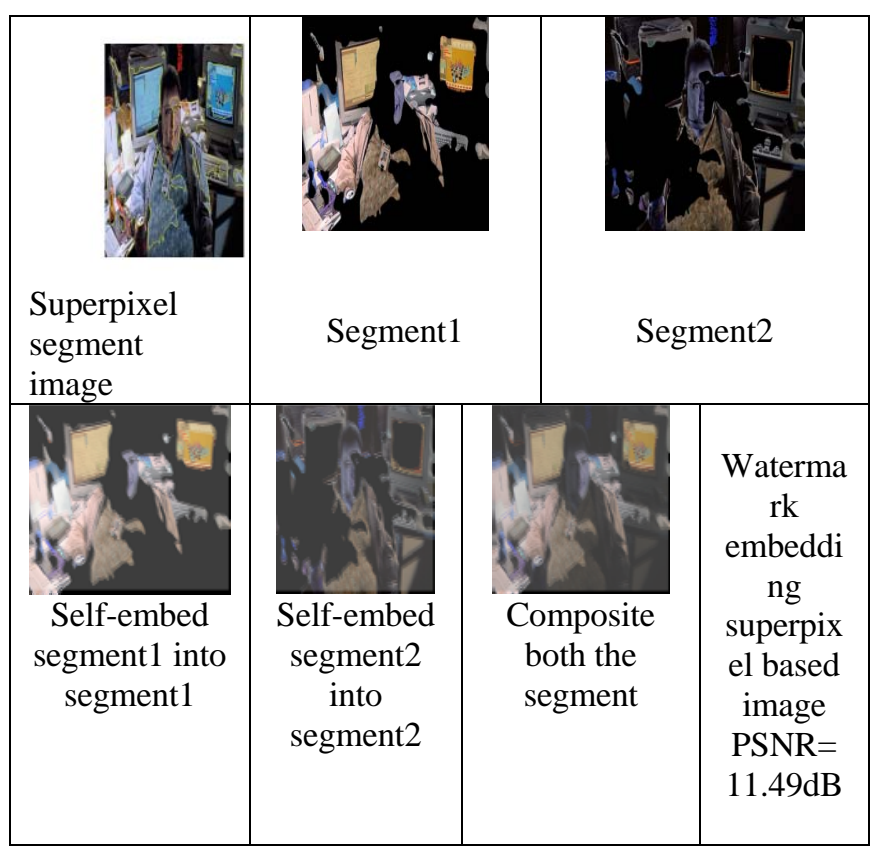

Figure3. Self-embedding watermark into individual Superpixel segments:

The analysis of the measure PSNR of the self-embedding watermark segments of composite image reveals that the PSNR value is 11.49DB which is less then acceptable level. This suggests that by try and error methods different approaches of embedment is to be applied so that PSNR value comes closer to acceptable level. 
The extension of the work is carried out with the application of SVD based watermarking embedding method. In this case PSNR is measure. This approach is indicating that increase the value of PSNR 24.67DB which is near about acceptable range. Extraction of watermark from all image segments of Superpixel image. PSNR value of extracted watermark from image segment is $34.69 \mathrm{DB}$.

The analysis of the measure PSNR of the SVD based Superpixel image reveals that this embedding approach is

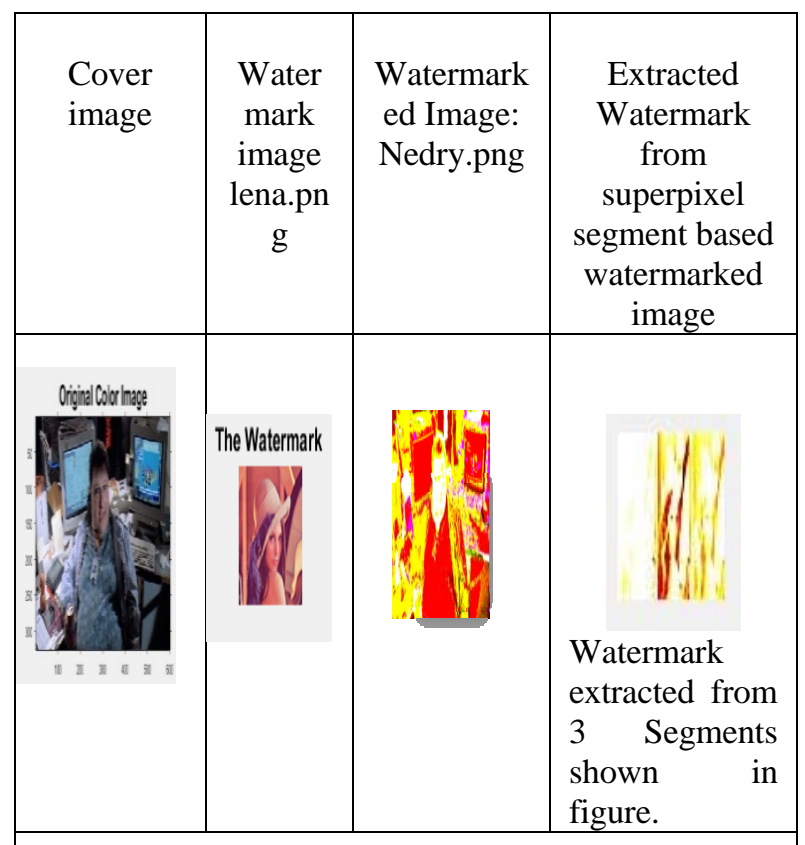

Watermark embedding by SVD in superpixel based image PSNR=14.546239dB

Original Watermark/Extracted Watermark from superpixel SVD based image PSNR=31.578620dB $(\mathrm{alfa}=1)$

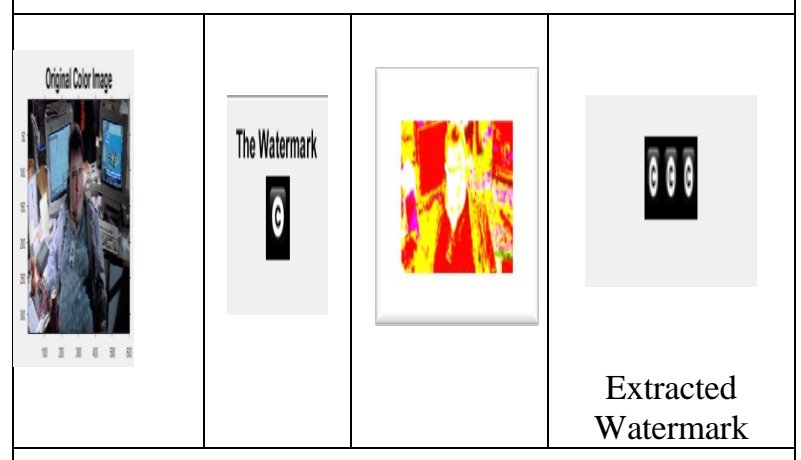

Watermark embedding by SVD in superpixel based image $\mathrm{PSNR}=24.670255 \mathrm{~dB}$

Original Watermark/Extracted Watermark from superpixel SVD based image PSNR $=34.695485 \mathrm{dBalfa}=0.08$

Figure.4. Superpixel SVD based image watermarking:

Comparatively suitable for making authenticate image. Extract watermark from each segment of watermarked image. Experimental results show that quality of Superpixel-SVD based watermarking scheme is better compared to selfembedding and segment watermarked methods.

\section{EVALUATION PARAMETER}

Mean Square Error (MSE) and Peak Signal to Noise Ratio (PSNR) obtains the result for reliability of watermarked image. Here OI is the original cover image and RI is the reconstructed image (Watermarked Image)

MSE

$$
=\frac{1}{M N V} \sum_{i=0}^{M-1} \sum_{j=0}^{M-1}[|O L-R I|]^{2}
$$

PSNR=

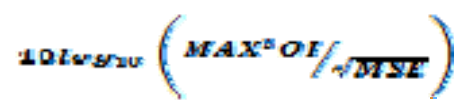

\section{CONCLUSION}

In this paper, we have presented various approaches of watermarking embedding techniques based on Superpixel segmentation for authentication and reliability of image .In this paper we have found Superpixel segment based watermarking methods using SVD transformation could achieve optimum PSNR value of 24.59DB.The segmentation of image which is input to Superpixel approach is optimize by segmentation based method compare to regular block based methods. Further it has been found that selfembedment is also indicating higher level of accuracy compare to external digital watermark image. This work can be extended for enhancing PSNR value for color image authentication in segment level embedding, tamper detection and localization.

\section{REFERENCES}

[1] Xiumei Qiao, Rongrong Ni and Yao Zhao. “ SuperpixelBased Watermarking Scheme for Image Authentication and Recovery" Digital-Forensics and Watermarking Volume 9023 of the series Lecture Notes in Computer Science pp 160-173

[2] R Achanta. "SLIC Superpixels” EPFL Technical Report 149300, 2010, Pg.no.1-15.

[3] Radhakrishna Achanta, Appu Shaji, Kevin Smith, Aurelien Lucchi, Pascal Fua, and Sabine Susstrunk. "SLIC Superpixels Compared to State-of-the-art Superpixel Methods”JOURNAL OF LATEX CLASS FILES, VOL. 6, NO. 1, DECEMBER 2011

[4] Clara Cruz-Ramos, Rogelio Reyes-Reyes, Mariko NakanoMiyatake,and Héctor Pérez-Meana"Image Authentication Scheme Based on Self-embedding Watermarking” SpringerVerlag Berlin Heidelberg 2009 pages 1005-1012

[5] Yan Xing , Jieqing Tan,” A Color Watermarking Scheme Based on Block-SVD and Arnold Transformation” IEEE Digital Media and its Application in Museum \& Heritages, Second Workshop on Dec 2007 pages 3-8

[6] Clara Cruz, Rogelio Reyes, Mariko Nakano*, Héctor Pérez,” Semi-fragile watermarking based content image authentication scheme "Rev. Fac. Ing. Univ. Antioquia N. ${ }^{\circ}$ 56 pp. 160-169. December, 2010

[7] Hong- Jie He, Jia-ShuZhang and FanChen.” Adjacent-block based statistical detection method for self-embedding watermarking techniques” Elsevier -Signal Processing 89 (2009) pp1557-1566 